> Les réseaux sociaux pour les chercheurs s'imposent au fil du temps auprès des communautés académiques, notamment grâce aux fonctionnalités de dépôt et de partage d'articles qu'ils proposent. L'objet de cette étude est d'évaluer la proportion, la typologie (pre-print, version auteur non expertisée ; post-print auteur/éditeur, versions auteur et éditeur expertisées définitives) et la légalité des textes intégraux d'articles qui y sont déposés par les chercheurs, à partir d'un échantillon de 1500 articles publiés entre 2013 et 2015 sélectionnés aléatoirement dans PubMed, en prenant pour exemple ResearchGate qui est très largement utilisé par la communauté médicale et biologique. À titre de comparaison, l'accès au texte intégral des 1500 articles via PubMed et PubMedCentral a également été évalué, mettant en évidence le rôle important que joue ResearchGate dans la fourniture de textes intégraux d'articles en biologie-médecine. Cet article met également en perspective le positionnement des réseaux sociaux par rapport aux archives ouvertes et au libre accès. <

De la même façon que les réseaux sociaux généralistes ou professionnels comme Facebook ou LinkedIn, ont trouvé leur place auprès du grand public depuis une dizaine d'années, les réseaux sociaux pour les chercheurs creusent leur sillon auprès des communautés universitaires et de recherche [1]. Si à la fin des années 2000 , ces services étaient encore en cours de structuration et leur usage peu répandu [2], ce n'est plus le cas actuellement : en 2014, $42 \%$ des chercheurs français utilisaient soit Academia ${ }^{1}$ soit ResearchGate (RG) ${ }^{2}$, qui comptent, respectivement, plus de 43 millions et 11 millions de comptes au niveau mondial [3]. De nombreuses études ont, par ailleurs, mis en évidence le leadership

${ }^{1}$ http://wwww.academia.edu

${ }^{2}$ https://www.researchgate.net/

\section{Réseaux sociaux \\ académiques \\ et diffusion \\ de la production \\ scientifique \\ des chercheurs en \\ biologie/médecine}

L'exemple

de ResearchGate

Christophe Boudry ${ }^{1-3}$, Aline Bouchard ${ }^{2}$

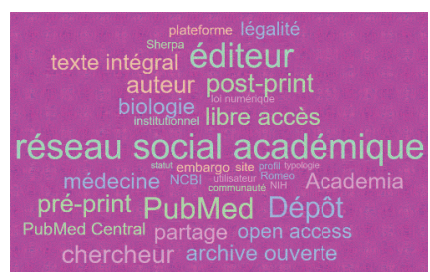

${ }^{1}$ Normandie Université, UNICAEN, Média Normandie. Esplanade de la Paix, 14032 Caen Cedex 5, France.

${ }^{2}$ Unité régionale de formation à l'information scientifique et technique (URFIST), દ́cole nationale des Chartes, Université de recherche Paris Sciences et Lettres, 17 rue des Bernardins, 75005 Paris, France.

${ }^{3}$ Laboratoire Dispositifs d'inforde ces deux plateformes [4-7]. Un tel développement s'explique en partie par leur utilisation des codes du web $2.0^{3}[8,9](\rightarrow)$, à commencer par la facilité d'utilisation et la gratuité, mais surtout par leur insertion dans l'écosystème académique. Généralement créés par d'anciens chercheurs qui connaissent bien le monde académique, ces services mettent au cœur de leur système le besoin d'interactions des mation et de communication à I'ère numérique (DICEN), عA7339, CNAM, 292 rue Saint Martin 75003 Paris, France.

christophe.boudry

@enc-sorbonne.fr chercheurs pour construire leurs communautés. ResearchGate en est un bon exemple : ouvert en 2008 par deux virologues et un informaticien, au contraire d'Academia qui est plutôt orienté en sciences humaines et sociales [10], ce réseau plutôt orienté sciences, techniques et médecine $[5,11]$, propose, outre des fonctionnalités de réseautage social (profil personnel, abonnements, questions-réponses), des fonctionnalités de dépôt et de partage de documents, au plus près donc des pratiques des chercheurs. On ne s'étonnera pas cependant que ce soient les fonctionnalités centrées sur l'utilisateur qui soient les plus utilisées, au détriment des fonctions vraiment sociales [6] : en ces périodes de concurrence et de compétition accrue, comme de précarisation de l'em-

${ }^{3}$ Web 2.0 désigne l'ensemble des techniques et fonctionnalités caractérisé par plus de simplicité et d'interactivité que le web initial (www, World Wide Web). II concerne en particulier les interfaces et les échanges permettant sans connaissances techniques d'utiliser de nouvelles fonctionnalités. 
ploi scientifique, les réseaux sociaux s'insèrent parfaitement dans les mécanismes de réputation et de visibilité qui voient le fameux «publish or perish » être progressivement complété par «be visible or vanish » [12]. Si les réseaux sociaux académiques (RSA) sont en premier lieu utilisés comme outil de profil et de visibilité, le partage de documents sur ces plateformes semble également être entré dans les pratiques académiques [5], dans la continuité du mouvement Open Access. Ce mouvement, qui a débuté dans les années 1990, s'est concrétisé dans deux directions; d'une part, par la mise en place d'archives ouvertes institutionnelles nationales (comme HAL Inserm et HAL CNRS, hyper archive en ligne) ou internationales (comme PubMed Central), afin que les chercheurs puissent, dans la mesure des restrictions imposées par certains éditeurs, auto-archiver leur production scientifique, afin d'en rendre le texte intégral librement accessible; d'autre part, par l'émergence de revues en libre accès, dont le texte intégral des articles est accessible librement. L'absence d'abonnement et donc du revenu y afférent pour leurs éditeurs est alors, en règle générale, compensée par des frais supplémentaires (article processing charge, APC) supportés par les auteurs [13].

Tous ces éléments amènent naturellement à s'interroger quant à la nature des textes intégraux (TI) d'articles déposés sur les réseaux sociaux académiques (RSA). L'objectif de cette revue est donc d'explorer les dépôts de textes intégraux, en particulier sur ResearchGate, d'un point de vue du nombre, de leur typologie (pre-print, post-print auteur ou éditeur) et de la légalité de leur exposition en regard des prescriptions des éditeurs, ce qui à notre connaissance n'a jamais été réalisé auparavant. Le réseau social académique choisi pour cette étude a été ResearchGate, car c'est celui qui est le plus utilisé dans le domaine de la bio-médecine [5], cadre de notre étude.

\section{Méthodologie employée}

La recherche des articles inclus dans cette étude a été effectuée en juin 2016, en utilisant la base de données PubMed $^{4}$ [14] $(\rightarrow)$. Un tirage aléatoire d'articles (de type «Journal article » dans le champ «Publication Type ») présents dans PubMed a été effectué à partir du

$(\rightarrow)$ Voir le Dossier technique de C. Boudry et G. Bozet, $\mathrm{m} / \mathrm{s}$ $n^{\circ}$ 8-9, août-septembre 2004, page 804 numéro d'identification utilisé par PubMed (PMID pour PubMed identifier $)^{5}$, afin d'obtenir un total de 1500 articles s'échelonnant sur les dates de publication de 2013 à 2015 (soit 500 par année). Pour chaque article, les données ont été téléchargées depuis PubMed en extensible markup language (XML) et en hypertext markup language (HTML), avec des scripts développés spécifiquement en hypertext preprocessor language (PHP), et en utilisant les fonctionnalités entrez programming utilities du Centre américain pour les informations biotechnologiques (NCBI, national center for biotechnology information) comme pratiqué précédemment $[15,16]$. Les données ont ensuite été importées dans un tableur pour être analysées.

${ }^{4}$ https://www.ncbi.nlm.nih.gov/pubmed/

${ }^{5}$ Voir glossaire.
ResearchGate (RG) a été interrogé manuellement, à partir du titre de chacun des 1500 articles sélectionnés, afin de déterminer leur présence ou leur absence sur la plateforme, ainsi que la possibilité d'accéder à son texte intégral (TI). Seuls ont été pris en compte les textes déposés par leurs auteurs (et donc hébergés sur $R G$ ) et non ceux disponibles via un lien externe généré par RG ou indiqué par le chercheur lui-même (par exemple, vers une archive ouverte ou un site personnel). Chaque texte intégral a été visualisé, afin de vérifier s'il correspondait bien à la référence décrite sur RG, puis a été labellisé en pre-print, post-print auteur ou post-print éditeur. Afin de déterminer si la présence de chaque texte intégral sur ResearchGate était légale ou non, une recherche sur le site de l'éditeur à partir du DOI (digital object identifier) ou à partir des liens externes présents dans PubMed a été effectuée, pour savoir si le texte y était ou non accessible en libre accès. Le statut de chaque article et la possibilité de réutilisation légale ou non de son texte intégral, notamment via la présence d'une licence creative commons, a été évalué. De plus, le site SHERPA/RoMEO6 a été systématiquement interrogé, afin de connaître les possibilités et les conditions d'auto-archivage des pre-prints, post-prints auteur et post-prints éditeur de chaque article, et ce pour déterminer si la présence de ce texte sur ResearchGate était légale ou non. La date de publication a été considérée pour déterminer les éventuelles périodes d'embargo. L'existence d'un lien vers le texte intégral de chacun des 1500 articles étudiés a également été explorée via les fonctionnalités LinkOut de PubMed, pour déterminer le nombre d'articles dont le texte était disponible gratuitement en libre accès sur le site des éditeurs, à partir de PubMed. Enfin, la présence ou l'absence de chacun des 1500 articles étudiés sur l'archive ouverte de PubMed Central $(P M C)^{7}$, et donc de leur texte intégral, a également été évaluée.

\section{Les réseaux sociaux académiques : un mode d'accès « incontournable » aux publications scientifiques et à leurs textes intégraux?}

Durant les quatre premières années de l'existence de ResearchGate, 2,5 millions de documents ont été mis en ligne. Ce chiffre correspond désormais au nombre de documents déposés chaque mois sur cette plateforme [17]! Actuellement, ResearchGate et ses 100 millions de documents [18] serait la troisième base de données d'articles derrière Google Scholar et Web of Science,

${ }^{6}$ http://www.sherpa.ac.uk/romeo/index.php

${ }^{7}$ https://www.ncbi.nlm.nih.gov/pmc/ 


\section{GLOSSAIRE}

PMID (PubMed identifier) et DOI (digital object identifier) : il s'agit d'identifiants numériques du manuscrit publié qui permettent de le cibler dans différentes bases de données et, plus spécifiquement, dans PubMed pour le PMID.

HTML (hypertext markup language) : signifie langage de balisage d'hypertexte. Ce langage permet de réaliser de l'hypertexte à base d'une structure de balisage. Les balises permettent de mettre en forme le texte et de placer des éléments interactifs comme des liens, des images ou des animations.

XML (extensible markup language) : signifie langage de balisage extensible. C'est un langage informatique qui permet de décrire des données à l'aide de balises et de règles que l'on peut personnaliser, avec pour objectif principal l'échange de données. Ce langage est libre et un fichier XML peut être créé à partir d'un simple logiciel de traitement de texte.

PHP (hypertext preprocessor language) : ce langage informatique est principalement utilisé pour produire un site web dynamique. II est souvent associé à une base de données comme MySpL (my structured query language).

Fonctionnalités entrez programming utilities : ces utilitaires de programmation sont un ensemble d'API (application programming interface) qui permettent d'interroger les bases de données du Centre américain pour les informations biotechnologiques (NCBI, national center for biotechnology information), dont PubMed, à partir de programmes développés par les utilisateurs, notamment afin de permettre à ceux-ci d'en extraire des lots de données importants, sans encombrer les interfaces d'interrogation publiques.

Fonctionnalités LinkOut : ces services permettent de lier les ressources des bases de données du Centre américain pour les informations biotechnologiques (NCBI, national center for biotechnology information) à d'autres services externes au NCBI, avec pour objectif de les compléter. Licences creative commons: constituent un ensemble de licences régissant les conditions de réutilisation et de distribution d'œuvres (multimedia, textes, etc.) et proposant des contrats-type pour leur mise à disposition en ligne.

Digital millenium copyright act : cette loi américaine a pour objectif de fournir un moyen de lutte contre les violations du droit d'auteur et vise à établir une législation de la propriété intellectuelle adaptée à l'ère numérique.

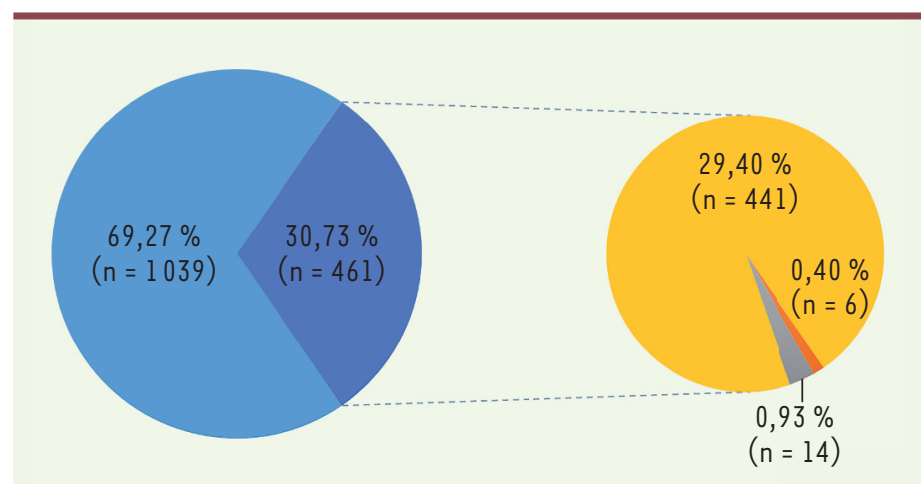

घI non présent $\approx$ Pre-print $₫$ Post-print auteur $\backsim$ Post-print éditeur qui, eux, comptent autour de 170 millions de références, et ce bien loin devant Academia (11 millions) [19] ; cela le placerait en deuxième place concernant le mode d'accès aux publications scientifiques derrière les accès institutionnels [20]. ResearchGate serait ainsi le site fournissant le plus de textes intégraux sur Google Scholar (plus d'l article sur 10) [21], devant le site du NCBI qui héberge notamment PubMed et PubMed Central.

Concernant l'échantillon que nous avons étudié, les textes intégraux (TI) de 461 des 1500 articles (30,7\%) étaient disponibles directement sur ResearchGate (Figure 1), chiffre à comparer à celui de l'étude de Orduña-Malea et al. [19], qui a évalué approximativement à $23,5 \%$ le pourcentage d'articles ayant un texte intégral sur cette plateforme, toutes disciplines confondues. Sur ces 461 articles, la très grande majorité était des versions post-prints d'éditeur (441 soit $95,7 \%$ ). Le chiffre de $30,7 \%$ est particulièrement important, car il ne concerne, rappelons-le, que les textes intégraux déposés par le chercheur et ne prend pas en compte les simples liens vers ceux hébergés à l'extérieur de la plateforme. À titre de comparaison, sur le même échantillon, il faut noter que via PubMed, seuls 353 articles (soit 23,5\% des articles étudiés) proposaient un accès au texte intégral sur le site de l'éditeur via la fonctionnalité LinkOut, et que l'archive ouverte PMC fournissait un accès au texte de 483 articles $(32,2 \%)$, soit à peine plus que ResearchGate. Comme le montre la Figure 2, qui détaille les pourcentages de textes intégraux accessibles via ResearchGate (RG), PubMed et l'archive ouverte PMC, pour les années 2013 à 2015, ResearchGate permet d'accéder, pour les articles publiés en 2014 et 2015, à plus de textes intégraux (TI) que ne le permettent respectivement PubMed et PubMed Central.

L'augmentation du nombre de ces textes intégraux disponibles, entre 2013 et 2014 , peut s'expliquer, d'une part, par une augmentation des articles publiés en libre accès et donc disponibles via PubMed et PubMed Central [22], et, d'autre part, pour ResearchGate, par une augmentation du nombre de ses usagers, qui augmente mécaniquement le nombre de textes intégraux déposés. Ce phénomène est d'autant plus important que les chercheurs ont tendance à augmenter leurs pratiques de partage, encouragés par le fait qu'ils trouvent des

Figure 1. Pourcentage d'articles dont le TI (texte intégral) est accessible directement sur RG (ResearchGate) (années 20132015). Détail par type de dépôt (pre-print, post-print auteur, post-print éditeur). 


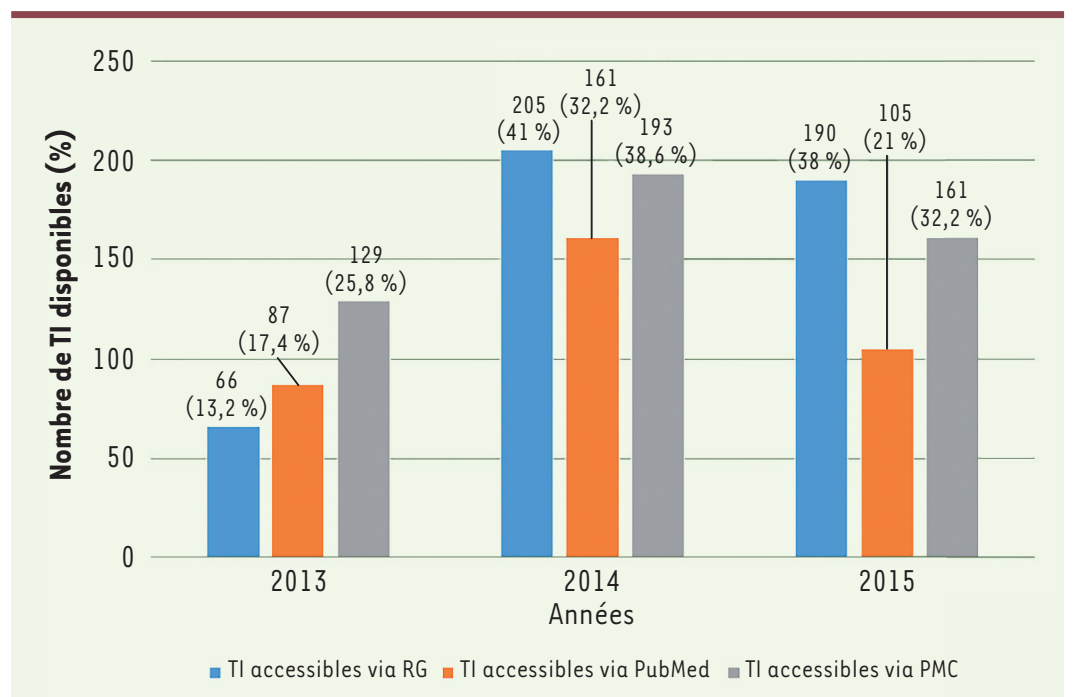

Figure 2. Nombre et pourcentage de TI (textes intégraux) accessibles via ResearchGate (RG), PubMed et PubMed Central, publiés de 2013 à 2015.

publications en textes intégraux sur ResearchGate. Ils déposent alors les leurs afin de permettre à leurs collègues d'en découvrir à leur tour, mais également pour augmenter la visibilité de leurs recherches. La diminution généralisée entre les années 2014 et 2015 du nombre de textes intégraux disponibles semble être la conséquence directe des embargos imposés par certains éditeurs [23], empêchant ainsi la mise à disposition immédiate d'un certain nombre d'entre-eux, cela de façon certaine pour PubMed et PubMed Central, de manière plus hypothétique pour ResearchGate. En effet, pour PubMed, l'accès aux textes intégraux est possible par des liens vers les sites des éditeurs qui respectent forcément leurs propres embargos. Le problème est identique pour PubMed Central où les dépôts sont effectués par les éditeurs eux-mêmes, si les NIH (National Institutes of Health) ont participé financièrement à l'étude et/ou si la revue est en libre accès, ou par les auteurs qui sont alors très encadrés lors du dépôt, afin de respecter les prescriptions des éditeurs. Cela n'est pas le cas pour ResearchGate où les auteurs sont beaucoup moins encadrés lors du dépôt. La baisse entre 2014 et 2015 du nombre de textes intégraux disponibles sur ResearchGate est vraisemblablement la conséquence du contrôle juridique accru que les éditeurs exercent désormais sur les réseaux sociaux académiques [24].

\section{Partager des articles en texte intégral sur ResearchGate : des questions de légalité}

De manière évidente, ResearchGate participe de l'open science, puisque les chercheurs du monde entier, quelles que soient leurs disciplines et leurs langues et indépendamment des moyens financiers de leurs institutions de rattachement, peuvent profiter de ce nouveau canal pour rechercher et accéder gratuitement aux articles scientifiques [20], à commencer par les articles récents [5]. L'initiative conjointe de plusieurs éditeurs, comme Elsevier, Springer, Nature, Wiley ou encore Taylor \& Francis, d'établir en 2015 des «Voluntary principles for article sharing on scholarly collaboration networks » [25], attire cependant l'attention sur la légalité de la majorité des dépôts d'articles dans leurs versions définitives (post-prints auteurs/éditeurs). Rappelons, en effet, que l'auto-archivage de ce type de documents est soumis au cadre contractuel établi entre les auteurs et leur éditeur. Or si, déjà, l'autoarchivage dans une archive ouverte ou sur un site personnel n'est pas libre [26], c'est $a$ fortiori le cas pour les réseaux sociaux académiques, sociétés commerciales qui parfois s'attribuent des licences de ré-exploitation sur les contenus déposés (à titre d'exemple, on pourra se reporter aux conditions d'utilisations d'Academia) [27]. De plus en plus d'éditeurs font d'ailleurs une distinction claire entre la politique d'auto-archivage autorisée sur des services not for profit et les réseaux sociaux académiques. Les autorisations de réutilisation des textes intégraux par les auteurs varient également en fonction du statut de l'article (pre-print, post-print auteur ou éditeur) et d'un éventuel embargo imposé par l'éditeur, et peuvent être consultées, comme nous l'avons fait, par exemple, sur le site SHERPA/RoMEO.

Concernant l'échantillon que nous avons étudié, près de la moitié des textes intégraux disponibles sur Research Gate (225 sur 461 soit $48,8 \%$ ) étaient exposés de manière illégale par rapport aux préconisations de l'éditeur sur leur mise à disposition par les auteurs. Ces résultats rejoignent ceux d'une étude réalisée par le Research Information Network (RIN) qui, à partir de deux échantillons (I'un regroupant des articles écrits par des auteurs provenant du monde entier et l'autre issus d'articles écrits uniquement par des chercheurs du Royaume-Uni), a estimé que les réseaux sociaux académiques, et principalement ResearchGate, étaient à l'origine de $70 \%$ des dépôts illégaux d'articles sur internet [28]. Sur les 225 dépôts illégaux, 223 (99,1\%) étaient des post-prints éditeur, 2 des post-prints auteur $(0,9 \%)$, et aucun pre-print n'était présent. Notons que ces résultats sont équivalents à ceux obtenus dans l'étude du Research Information Network [28], qui a estimé que $90 \%$ des dépôts illégaux retrouvés sur internet sont des post-prints éditeur, déposés principalement sur les réseaux sociaux académiques. Cette utilisation majoritaire de la version post-print éditeur se justifie par les pratiques mêmes des chercheurs : plus de trois universitaires sur quatre $(77 \%)$ préfèrent en effet partager la version éditeur de leurs publications 


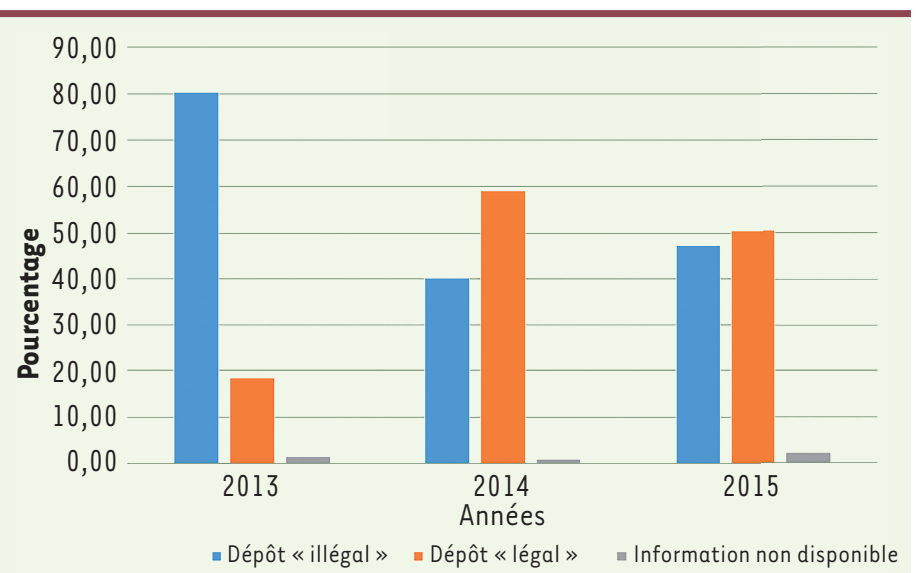

Figure 3. Statut des articles dont le TI (texte intégral) est accessible sur RG (ResearchGate) (dépôt légal/illégal), publiés de 2013 à 2015. «Information non disponible » signifie que l'information concernant la politique d'auto-archivage du journal n'était pas disponible sur SHERPA/RoMEO (Ungraded journal)

[29], version d'usage permettant notamment de pouvoir citer correctement un article, car présentant toutes les données nécessaires à cela (volume, pagination, titre de la revue, etc.).

Comme le montre la Figure 3 , le pourcentage de dépôts illégaux était maximal pour les publications en 2013 , représentant $80,3 \%$ des dépôts, pour s'établir à $47,37 \%$ en 2015 . Cette baisse du pourcentage des dépôts illégaux au fur et à mesure du temps est probablement à mettre à l'actif de l'augmentation concomitante du nombre d'articles publiés en libre accès sur la même période, dont les conditions de réexploitation du texte intégral sont en général moins contraignantes. Cette baisse est cependant contradictoire par rapport à l'existence de périodes d'embargo qui s'appliquent sur certains articles non publiés en libre accès et qui limitent leur ré-exploitation, sachant que les périodes d'embargo ont classiquement des durées comprises entre 6 et 12 mois [30].

Une proportion aussi importante de dépôts illégaux sur ResearchGate peut s'expliquer par le fait que les réseaux sociaux académiques

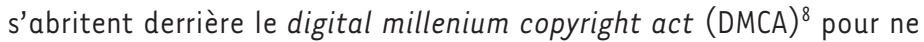
pas vérifier la légalité des dépôts pratiqués sur leurs sites. De fait, selon les principes du DMCA, la responsabilité du dépôt est portée par l'utilisateur, les réseaux se considérant comme de simples plateformes de contenus sans responsabilité, à l'instar de YouTube [31]. Et si ResearchGate mentionne le site SHERPA/RoMEO lors du dépôt, c'est à titre purement informatif et sans aucun blocage technique. Ne pas vérifier la légalité des dépôts est une chose, pousser au dépôt en est cependant une autre. Or, dans ce domaine, la responsabilité de ResearchGate est claire à trois niveaux. D'abord, tout le discours de l'entreprise milite pour l'open science et le partage de la recherche, entretenant une subtile confusion avec l'open access, alors même que déposer sur ResearchGate n'équivaut pas à un dépôt sur une archive ouverte [32]. Ensuite, l'entreprise développe toute une série de

${ }^{8}$ https://www.gpo.gov/fdsys/pkg/PLAW-105pub/304/pdf/PLAW-105publ304.pdf services (open review, citations, réseaux) centrés sur les publications déposées, y compris en jouant sur l'ego du chercheur et sa recherche de visibilité, en proposant ses propres paramètres bibliométriques comme le ResearchGate score [4, 33]. Enfin, profitant du manque de temps et de compétences techniques des chercheurs, ResearchGate donne l'impression de ne pouvoir indiquer le lien vers une version en ligne de la publication qu'à la condition d'avoir déposé le fichier, ce qui n'est pas le cas.

\section{Conclusion}

L'analyse que nous proposons présente certaines limitations : le nombre d'articles étudiés est en effet limité, cela étant justifié par une vérification manuelle chronophage de leur présence effective sur ResearchGate et de leur statut légal par rapport aux prescriptions des éditeurs qui les publient. Malgré ces limitations, elle apporte un éclairage complémentaire par rapport aux études précédentes réalisées sur les réseaux sociaux académiques, notamment en ce qui concerne le nombre, la typologie et le statut légal des textes intégraux que ResearchGate héberge. Ces différentes études mettent en évidence que les réseaux sociaux académiques ne cessent de prendre de plus en plus d'importance dans le paysage de la recherche mondiale, les services proposés par ces plateformes ne cessant de se perfectionner, le nombre de leurs utilisateurs ne cessant de progresser, et le rôle qu'ils jouent dans la diffusion de la production scientifique des chercheurs ne cessant également de croître.

Dans le même temps, profitant de la dynamique du mouvement open access, les pouvoirs publics ont consenti de gros efforts afin de développer et mettre en place des plateformes institutionnelles d'archives ouvertes comme PubMed Central ou HAL (il existe 3636 archives ouvertes dans le monde, réparties dans 99 pays différents) [34] et d'en promouvoir l'utilisation à travers des mandats institutionnels (pas moins de 748 mandats répartis dans 61 pays existent au niveau mondial) [35]. À l'instar des réseaux sociaux académiques, de nombreux services institutionnels permettant à chacun de se créer un profil et un curriculum vitae numérique ont également été développés (voir par exemple pour la France https://cv.archives-ouvertes. $\mathrm{fr} /$ ). Ces efforts auraient pu inciter les chercheurs institutionnels à privilégier les dépôts d'articles sur ces plateformes et à les utiliser en priorité pour communiquer, ce qui n'est pas le cas. En effet, la prééminence de ResearchGate dans les usages académiques, à la fois pour la recherche d'informations [36] et pour le 
dépôt d'articles s'explique globalement par l'ignorance de l'existence des archives ouvertes et de leur fonctionnement, d'une part, et par les avantages que les chercheurs attribuent à ResearchGate, d'autre part (facilité d'utilisation globale et des dépôts en particulier, meilleure visibilité des articles) [37].

Il semble donc primordial de poursuivre les efforts consentis par les pouvoirs publics, via des actions de formation/information, afin que les communautés universitaires et de recherche appréhendent mieux ces enjeux de communication scientifique et d'identité numérique. L'environnement pour atteindre ces objectifs est d'autant plus favorable en France aujourd'hui que celle-ci a adopté en 2016 la « loi pour une République numérique », qui élargit significativement les possibilités de dépôt sur les archives ouvertes pour tout article publié à partir du 9 octobre 2016 et financé, au moins pour moitié, par des fonds publics [38]. $\diamond$

\section{SUMMARY}

Role of academic social networks in disseminating

the scientific production of researchers in biology/medicine:

the example of ResearchGate

Over time, academic social networks are more and more used by researchers, especially thanks to the possibilities of sharing articles they offer. The objective of the present study was to evaluate the proportion, the typology (pre-print, post-print author/publisher) and the legality of the full-text publications deposited by researchers on ResearchGate which is widely used by the medical and biological community, using a sample of 1,500 randomly selected articles in PubMed and published between 2013 and 2015. To compare, the access to the full-text of the 1500 articles via PubMed and PubMed Central has been assessed, putting into evidence the important role ResearchGate plays for providing full-texts of articles in biology/medicine. It also puts academic social networks into perspective in relation to open-access repositories and open access. $\diamond$

\section{LIENS D'INTÉRÊT}

Les auteurs déclarent n'avoir aucun lien d'intérêt concernant les données publiées dans cet article.

\section{RÉFÉRENCES}

1. Williams AE, Woodacre MA. The possibilities and perils of academic social networking sites. Online Inf Rev $2016 ; 40: 282-94$.

2. Gallezot G, Le Deuff 0. Chercheurs 2.0 ? Les Cahiers du numérique $2010 ; 5: 15-31$.

3. Réseaux sociaux de la recherche et Open Access. Perception des chercheurs. Etude exploratoire. Disponible sur <http://couperin.org/images/stories/openaire/Couperin_RSDR\%20et\%200A_ Etude\%20exploratoire_2014.pdf>.

4. Thelwall M, Kousha K. ResearchGate: Disseminating, Communicating, and Measuring Scholarship ? JASIST $2015 ; 66: 876-89$.

5. Thelwall M, Kousha K. ResearchGate articles: Age, discipline, audience size, and impact. JASIST $2016 ; 68: 468-79$.

6. Van Noorden R. Online collaboration: Scientists and the social network. Nature $2014 ; 512$ : 126-9.

7. Mikki S, Zygmuntowska M, Gjesdal ØL, et al. Digital presence of Norwegian scholars on academic network sites-Where and who are they? PLOS ONE $2015 ; 10$ : e 0142709.

8. Boudry C. «Biologie/médecine $2.0 »$ : état des lieux. Med Sci (Paris) $2012 ; 28: 653-8$.

9. Boudry C. Web 2.0 Applications in Medicine: Trends and Topics in the Literature. Med 2.02015 ; $4:$ :e2.

10. Thelwall M, Kousha K. Academia.edu: Social network or Academic Network ? JASIST 2014 ; 65 : 721-31.
11. Ortega JL. Disciplinary differences in the use of academic social networking sites. Online Inform Rev $2015 ; 39: 520-36$.

12. Doyle J, Cuthill M. Does "get visible or vanish" herald the end of "publish or perish" ? Higher Education Research \& Development 2015 ; 34 : 671-4.

13. Boudry C, Durand-Barthez M. Publications en libre accès en biologiemédecine : historique et état des lieux en 2016. Ethics, Medicine and Public Health $2017 ; 3: 168-80$.

14. Boudry C, Bozet G. Recherche bibliographique en biologie et en médecine: du bon usage de Medline Pubmed. Med Sci (Paris) 2004 ; $20: 804-7$.

15. Boudry C, Denion $\varepsilon$, Mortemousque $B$, et al. Trends and topics in eye disease research in PubMed from 2010 to 2014. PeerJ 2016 ; 4 : el557.

16. Boudry C, Chartron G. Availability of digital object identifiers in publications archived by PubMed. Scientometrics $2017 ; 110$ : 1453-69.

17. Virtual Networks, Open Science and Digital Revolutions. Disponible sur <http://lab.cccb.org/en/virtual-networks-open-science-and-digitalrevolutions/>.

18. ResearchGate fact sheet. Disponible sur <https://www.researchgate.net/ aboutus.AboutUsPress.downloadFile.html?name=rg_fact_sheet.pdf $>$

19. Orduña-Malea $\varepsilon$, Martín-Martín A, López-Cózar ED. ResearchGate como fuente de evaluación científica: desvelando sus aplicaciones bibliométricas. El profesional de la información 2016 ; 25 : 303-10.

20. Innovations in Scholarly Communication. Disponible sur <https://10linnovations.wordpress.com/〉.

21. Jamali HR, Nabavi M. Open access and sources of full-text articles in Google Scholar in different subject fields. Scientometrics $2015 ; 105: 1635-51$.

22. Vardakas KZ, Tsopanakis G, Poulopoulou A, et al. An analysis of factors contributing to PubMed's growth. J Informetr 2015 ; 9 : 592-617.

23. Björk B-C, Laakso M, Welling P, et al. Anatomy of green open access. JASIST $2014 ; 65: 237-50$.

24. Posting Your Latest Article? You Might Have to Take It Down - Wired Campus - Blogs - The Chronicle of Higher Education. Disponible sur <http://www. chronicle.com/blogs/wiredcampus/posting-your-latest-article-you-mighthave-to-take-it-down/48865>.

25. \#DeleteAcademicSocialNetworks ? Les réseaux sociaux académiques en 2016. Disponible sur 〈http://urfistinfo.hypotheses.org/3033>.

26. SHERPA/RoMEO - Publisher copyright policies \& self-archiving. Disponible sur <http://www.sherpa.ac.uk/romeo/index.php〉.

27. Academia.edu | Terms. Disponible sur 〈https://www.academia.edu/terms〉.

28. Research Information Network. Monitoring the Transition to Open Access. A report for the Universities UK Open Access Co-ordination Group. Disponible sur <https://www.acu.ac.uk/research-information-network/monitoringtransition-to-open-access>.

29. Get the infographic! "Beyond Downloads: How scholars save \& share articles". Disponible sur <https://libraryconnect.elsevier.com/articles/getinfographic-beyond-downloads-how-scholars-save-share-articles>.

30. Laakso M. Green open access policies of scholarly journal publishers: a study of what, when, and where self-archiving is allowed. Scientometrics 2013 ; $99:$ 475-94.

31. ResearchGate. Terms and Conditions. Disponible sur 〈https://www.researchgate.net/application.TermsAndConditions.html〉.

32. A social networking site is not an open access repository. Disponible sur <http://osc.universityofcalifornia.edu/2015/12/a-social-networking-siteis-not-an-open-access-repository/>.

33. Yu M-C, Wu Y-CJ, Alhalabi W, et al. ResearchGate: An effective altmetric indicator for active researchers? Comput Human Behav 2016 ; 55 : 1001-6.

34. ROAR. Disponible sur <http://roar.eprints.org/>.

35. ROARMAP. Disponible sur <http://roarmap.eprints.org/>.

36. Nicholas D, Boukacem-Zeghmouri C, Rodríguez-Bravo B, et al. Where and how early career researchers find scholarly information. Learned Publishing $2017 ; 30: 19-29$.

37. Borrego Á. The depositing habits of Spanish researchers: Institutional repositories versus ResearchGate. Learned Publishing 2017. doi:10.1002/ leap.1099.

38. Vos dépôts dans HAL : ce qui change avec la loi pour une République Numérique | CCSD. Disponible sur <https://www.ccsd.cnrs.fr/fr/2016/10/ vos-depots-dans-hal-ce-qui-change-avec-la-loi-pour-une-republiquenumerique/s.
TIRÉS À PART

C. Boudry 